\title{
ABHANDLUNGEN
}

\section{Von Rebellen zu Eliten. Aufstieg und Scheitern bewaffneter Gruppen nach dem Krieg}

\author{
Stephan Hensell / Felix Gerdes
}

\section{From rebels to elites. The rise and fall of armed groups after war}

Abstract: In many post-war contexts, former rebels pursue political careers and their advancement is part of the formation of new elites. However, while some rise to senior political positions, others have to be content with lower level positions or are even completely marginalized. The article outlines a theory of the emergence and reproduction of elite power in the social space of the post-war society to explain this variance. The central proposition is that rebel careers are mainly dependent on power resources which have been accumulated after the war and not during the conflict. Case studies of Liberia and Kosovo serve as a first plausibility probe of our thesis and explain the post-war political careers of former rebels in the two cases with the help of the theoretical conception.

Keywords: Rebels, political elites, post-conflict societies, Liberia, Kosovo

Schlagwörter: Rebellen, politische Eliten, Nachkriegsgesellschaft, Liberia, Kosovo

\section{Einleitung}

Die Integration von bewaffneten Gruppen zählt zu den zentralen Herausforderungen nach dem Krieg. ${ }^{1}$ Um zu verhindern, dass Rebellen oder Warlords ${ }^{2}$ ihren Kampf wiederaufnehmen, gilt es Wege zu finden, sie in das soziale, politische und ökonomische Leben der Nachkriegsgesellschaft zu integrieren. Eine erste Welle der Forschung hat diesen Integrationsprozess mit Blick auf die Entwaffnung und Demobilisierung von Kombattanten analysiert (Springer 2007; Muggah 2009; Dodouet et al. 2012). Die Literatur hierzu mit ihrem Fokus auf technisch-praktische Probleme und Implementierungsfragen blieb jedoch im Wesentlichen deskriptiv und policy-orientiert (Berdal u. Ucko 2009, S. 2). Die eigentliche politische Transformation wurde dagegen eher vernachlässigt. So bemerkt Jeroen de Zeeuw: „Despite the importance of the political transformation of nonstate

1 Dieser Beitrag basiert auf Forschungen im Rahmen eines von der DFG (2010-2012) geförderten Projektes mit dem Titel „Die Formierung politischer Eliten in Nachkriegsgesellschaften“ am Institut für Politikwissenschaft der Universität Hamburg.

2 Wir verwenden im Folgenden die Begriffe Rebellen und Warlords als Bezeichnung für nicht-staatliche Gewaltakteure synonym. Für eine Differenzierung und Typologisierung unterschiedlicher bewaffneter Gruppen vgl. Schneckener (2012). 
armed movements in the settlement of civil wars and in postwar democratization, surprisingly little is known about this process" (2008b, S. 2).

Eine zweite Welle der Forschung begann sich daher mit den Chancen und Hindernissen der Umwandlung bewaffneter Gruppen in politische Parteien zu beschäftigen (Söderberg Kovacs 2007; Manning 2008; de Zeeuw 2008a). Fragen der Parteibildung, der Wählerbasis und der Demokratisierung stehen hier im Mittelpunkt. Obgleich dieser Ansatz eine wichtige Erweiterung gegenüber der früheren sicherheitsbezogenen Diskussion ist, zeichnet auch er sich durch Einseitigkeiten aus. Denn hier geht es ausschließlich um die Frage, wie sich Rebellengruppen in Organisationen umwandeln. Dieser Fokus reduziert die politische Transformation von Rebellen auf die Parteipolitik und geht a priori von demokratischen Parteien als den entscheidenden Akteuren aus. Es sind jedoch auch andere Wege der Reintegration von Gewaltakteuren denkbar. Wie Mats Berdal und David Ucko anmerken: „Specifically, limiting the meaning of political reintegration to the formal creation of political parties for the purpose of partaking in democratic political processes may be overly restrictive" $(2009$, S. 6). In der Tat können ehemalige Rebellen im politischen Establishment der Nachkriegsgesellschaft als Präsident, Parlamentarier, Minister oder hoher Beamter vertreten sein, ohne dabei notwendig einer Partei anzugehören, die aus einer Rebellengruppe hervorgegangen ist. Die vielfältigen Karrierewege ehemaliger Gewaltakteure werden mit dem Fokus auf politische Parteien nur zum Teil erfasst.

Wir setzen daher einen anderen Schwerpunkt und fragen in einer erweiterten akteurszentrierten Perspektive nach der Transformation von Rebellen in politische Eliten. Eine elitentheoretische Perspektive erlaubt es grundsätzlich, auch andere Wege der Reintegration in den Blick zu bekommen, ohne dabei von einem vorgegebenen organisatorischen Ziel wie einer politischen Partei auszugehen. Sie erlaubt es darüber hinaus zu untersuchen, ob es ehemaligen Rebellen gelingt, sich mit anderen Elitefraktionen zu einer neuen politischen Klasse zu verbinden, sodass sich die Chancen der Errichtung stabiler und demokratischer Nachkriegsregime abschätzen lassen (Burton u. Higley 1987). Von den politischen Eliten in Nachkriegsgesellschaften wird viel erwartet. Zu ihren Aufgaben gehören laut Daniel Serwer und Patricia Thomson u. a. die Entwicklung einer gesellschaftspolitischen Zielsetzung, die Umsetzung von Friedensabkommen, das Management von Ressourcen, die Einbeziehung internationaler Akteure sowie das Konfliktmanagement mit möglichen „spoilern“ (Serwer u. Thomson 2007, S. 381-386). Über die Formierung dieser Eliten, die diese Aufgaben meistern sollen, wissen wir jedoch noch wenig.

Ziel des Textes ist es, etwas mehr Licht auf diesen Prozess zu werfen und die Transformation von Rebellengruppen als Prozess der Elitewerdung zu analysieren. Dabei stehen die politischen Karrieren von Rebellen im Mittelpunkt. Die Laufbahnen dieser Akteure zeichnen sich durch Besonderheiten aus, die sie von typischen Verlaufsmustern in etablierten politischen Systemen unterscheiden. Nachkriegskarrieren von Rebellen sind diskontinuierlich verlaufende Prozesse, die weniger durch bestimmte Organisationen kanalisiert werden und stattdessen durch den unmittelbaren Sprung in Positionen gekennzeichnet sind. Darüber hinaus unterliegen Rebellenkarrieren dem ambivalenten Effekt der Gewalt, die diese 
Karrieren einerseits erst ermöglicht, aber andererseits stets überschattet (Schlichte 2009). Die Gewaltexpertise und die Kontrolle gewaltkompetenter Organisationen sind ein entscheidender Grund, warum Rebellen überhaupt Machtpositionen erringen, die ihnen Chancen auf einen politischen Aufstieg nach dem Krieg eröffnen. Die Gewalt wirft jedoch zugleich auch lange Schatten. Rebellen, die nach dem Krieg zu Politikern werden, müssen stets befürchten von ihrer Gewaltvergangenheit eingeholt zu werden und für Kriegsverbrechen und Menschenrechtsverletzungen zur Rechenschaft gezogen zu werden. Die de-legitimierenden Effekte der Gewalt können Karrieren auch wieder zunichtemachen, wie das Beispiel von Charles Taylor in Liberia oder Ramush Haradinaj im Kosovo belegen. Dies ist jedoch nicht oft der Fall. Stattdessen sind die Karrieren von Rebellen zumeist Regelmäßigkeiten unterworfen, die der Logik politischer Machtbildung in der Nachkriegsgesellschaft folgen. Sie zu skizzieren ist das Ziel des vorliegenden Beitrages.

Dem Aufsatz liegen folgende Fragestellungen zugrunde: Warum sind die politischen Karrieren von Gewaltakteuren nach dem Krieg so unterschiedlich? Warum gelingt es einigen Rebellen nach dem Krieg in höchste staatliche Ämter aufzusteigen, während sich andere in subalternen Positionen wiederfinden? Was entscheidet über ihren Erfolg oder Misserfolg in der Nachkriegsgesellschaft? ${ }^{3}$ Um diese Fragen zu beantworten und die beobachtbare Varianz zu erklären, sind etablierte Theorien politischer Eliten wenig hilfreich, da sie überwiegend von institutionell vorgeprägten Karrierewegen in funktional differenzierten Gesellschaften ausgehen und gewaltsame Regimewechsel als Modus des Elitenwandels ausschließen. Für die Analyse der Eliten in Nachkriegsgesellschaften, in denen die Institutionen schwach und die Polarisierung stark ist, sind diese Ansätze nur bedingt brauchbar. Wir streben daher einen eigenen theoretischen Entwurf an, der auf die soziale Mobilität von Gewaltakteuren fokussiert und der es erlaubt, die Genese und Reproduktion von Elitenmacht nach dem Krieg begrifflich zu fassen und zu erklären. Dieser Entwurf beansprucht Geltung für alle diejenigen Nachkriegsgesellschaften, in denen innerstaatliche Kriege durch Verhandlungslösungen oder Rebellensieg beendet wurden und folglich Rebellen Elitepositionen einnehmen konnten. ${ }^{4}$

Das Ziel des Textes ist es, eine Theorie der Elitewerdung beziehungsweise der politischen Karrieren von Rebellen zu umreißen und empirisch zu plausibilisieren. Wir definieren Eliten dabei als „persons who are able, by virtue of their strategic positions in powerful organizations, to affect national political outcomes regularly and substantially" (Field et al. 1990, S. 152). Einen geeigneten Ansatzpunkt für dieses Vorhaben liefert die Theorie der Praxis von Pierre Bourdieu mit den zentralen Kategorien Feld, Kapital und Habitus. Der Soziologe Bourdieu steht in einer Reihe mit den Klassikern der Elitesoziologie wie Gaetano Mosca, Vilfredo Pareto und Robert Michels. Im Gegensatz zu diesen Gründervätern stellt die Theorie

3 Als Erfolg verstehen wir das sukzessive Durchlaufen von unterschiedlichen Positionen und die Einnahme einer Elitestellung (siehe Kapitel 2.4).

4 Von 1945 bis 2011 sind weltweit 89 Kriege in dieser Form beendet worden. Quelle: Datenbank der Arbeitsgemeinschaft Kriegsursachenforschung (AKUF) an der Universität Hamburg (www.akuf.de). 
Bourdieus, die sich ausgiebig und durchgängig mit gesellschaftlichen Eliten beschäftigt hat, jedoch einen systematischeren und theoretisch konsistenteren Ansatz dar (Hartmann 2008, S. 37-41, 84-108; von Beyme 1992, S. 218-242). Eine bruchlose Anwendung der Theorie der Praxis ist jedoch nicht beabsichtigt. Stattdessen soll für das skizzierte Unterfangen vor allem der erweitere Kapitalbegriff Bourdieus nutzbar gemacht werden. Ferner spielt das von Robert Merton entwickelte Konzept der Opportunitätsstruktur (Merton 1996), das die strukturellen Zwänge der Handlungssituation von Akteuren in den Mittelpunkt stellt, eine zentrale Rolle.

Im ersten Kapitel umreißen wir mit dem sozialen Raum der Nachkriegsgesellschaft, dem Kapital der Eliten und der Opportunitätsstruktur die wesentlichen Elemente der Theorie und formulieren diese anhand eines Prozessmodells, das unterschiedliche Stufen von Rebellenkarrieren unterscheidet, weiter aus. Hieraus leiten wir die folgende These ab: Rebellenkarrieren sind in erster Linie von Machtmitteln abhängig, die nach dem Konflikt gewonnen werden und sich weniger einer Akkumulation im Krieg verdanken. Eine wesentliche Rolle spielen dabei der frühe Zugang zum Staat und die Erschließung von Einkommensquellen. Langfristig entscheidend für die Einnahme einer Eliteposition ist jedoch die Konversion dieser ökonomischen Ressourcen in neue vertikale und horizontale Bündnisse mit Klientelgruppen und anderen Elitefraktionen. Im zweiten Kapitel sollen Fallstudien zu Liberia und Kosovo diese These einem ersten Plausibilitätstest unterziehen und die Nachkriegskarrieren von Rebellen mit Hilfe der Theorie erklären. Die Fallstudien dienen zugleich dazu, die Fruchtbarkeit der theoretischen Konstruktionen am empirischen Material zu entfalten. Im Fazit werden die empirischen Befunde vergleichend diskutiert und es wird aufgezeigt, welche Implikation sich hieraus für internationale Akteure und die Demokratisierung von politischen Regimen nach dem Krieg ergeben.

\section{Von Rebellen zu Eliten in der Nachkriegsgesellschaft}

Kriege stoßen Dynamiken sozialer Mobilität und damit einen relativen Auf- und Abstieg sozialer Gruppen und Individuen an. Sie können, abhängig von ihrer räumlichen und zeitlichen Ausdehnung, mehr oder weniger tiefgreifende Veränderungen der sozialen Schichtung einer Gesellschaft und ihrer Elitenstruktur bewirken, die auch die Nachkriegszeit bestimmen. ${ }^{5}$ Rebellengruppen sind ein wesentlicher Bestandteil dieser Dynamik. Bereits im Krieg formieren sie sich als Gegeneliten, die politische Organisationen und Territorien kontrollieren. ${ }^{6}$ Gelingt es ihnen, durch militärische Erfolge Verhandlungslösungen herbeizuführen oder gar einen Sieg zu erringen, so eröffnet ihnen die politische Ausgestaltung der Nachkriegsordnung auch Chancen auf politische Karrieren. Diese Chancen eröffnen sich jedoch nicht allen Mitgliedern der Rebellengruppen.

5 Vgl. schon Sorokin (1927, S. 466-472) und aus der neueren Literatur Schlichte (2004); Malešeivić (2010, S. 237-274).

6 Oft reichen die Anfänge des Aufstiegs dieser Gegeneliten jedoch in die Zeit vor dem Krieg zurück. 
Bewaffnete Gruppen lassen sich nach Organisationsformen unterscheiden, die von losen Warlordfigurationen bis hin zu regelrechten Rebellenstaaten reichen (Bakonyi et al. 2006). Abhängig von ihrer Größe und Institutionalisierung bilden Rebellengruppen interne Stratifizierungen und organisatorische Hierarchien aus. Klaus Schlichte unterscheidet diesbezüglich zwischen Anführern, Stab und Gefolgschaft (Schlichte 2009, S. 32-38). Die biografischen Charakteristika von Anführern und Mitgliedern des Verwaltungsstabs bewaffneter Gruppen verdeutlichen die Bedeutung akademischer Ausbildung sowie die professionelle Erfahrung als Oppositionspolitiker und zum Teil auch als Mitglied der politischen Klasse (Malthaner 2007, S. 17-20; Schlichte 2009, S. 35). Zugleich bestehen in der Regel große sozio-ökonomische Differenzen zwischen den Anführen und dem Verwaltungsstab auf der einen Seite und der Gefolgschaft auf der anderen Seite (Schlichte 2009, S. 38). In innerstaatlichen Kriegen haben typischerweise die Führungsriege und Offiziersränge bewaffneter Gruppen die besten Aussichten nach dem Krieg hohe Positionen zu bekleiden. Die Frage, wer nach dem Krieg integriert wird und wer nicht, wird jedoch im Krieg nicht entschieden. Ob und inwiefern eine Fortsetzung von Karrieren im Nachkriegskontext möglich ist, hängt von den spezifischen Bedingungen der Nachkriegsgesellschaft ab.

\subsection{Der soziale Raum der Nachkriegsgesellschaft}

Ausgangspunkt für die folgende Skizze ist die Überlegung, dass sich zeitgenössische Nachkriegsgesellschaften als eine besondere Gesellschaftsform abgrenzen lassen und in Anlehnung an Pierre Bourdieus Theorie als „sozialer Raum“ konzeptionalisiert werden können (Schlichte 2004, S. 196-198). ${ }^{7}$ In der Theorie Bourdieus erlaubt das Modell des sozialen Raumes einen umfassenden Blick auf die Gesamtgesellschaft und ihre Klassenverhältnisse, wobei anhand der Kriterien Kapitalvolumen, Kapitalstruktur und soziale Laufbahn objektive soziale Positionen innerhalb dieses Raumes bestimmt werden können (Bourdieu 1998, S. 1327). Bezogen auf Nachkriegsgesellschaften lässt sich von einem sozialen Raum sprechen, in welchem sich für die Akteure Chancen sozialer Mobilität und des Aufstiegs eröffnen. Dies betrifft siegreiche Rebellenführer ebenso wie Profiteure und Unternehmer des Krieges, Vertreter der sozialen Opposition oder Anführer von Exilgruppen. Zugleich sind diese Akteure der Eigenlogik des politischen Feldes ausgesetzt, das sich als ein Handlungsfeld innerhalb des sozialen Raumes abgrenzen lässt und spezifische Praxisformen aufweist (Bourdieu 2000).

Max Weber zufolge lässt sich die Eigengesetzlichkeit der politischen Sphäre an der Verberuflichung politischer Karrieren festmachen (Borchert 2003, S. 89). Der Betriebscharakter der Politik bedingt das Aufkommen des Berufspolitikers, der zum einen in den ausschließlichen Dienst für die Politik tritt und zum anderen danach strebt, aus der Politik eine dauernde Einnahmequelle zu machen (Weber

7 Obschon die Forschung mittlerweile eine unübersehbare Literatur zu zahlreichen Einzelaspekten von Nachkriegskontexten produziert hat, mangelt es an Arbeiten, die Nachkriegsgesellschaften als eine spezifische Gesellschaftsform fassen. Vgl. für erste Ansätze in diese Richtung Brewer (2010, S. 16-28) und Bonacker et al. (2010). 
1988, S. 512-513). Rebellen, die eine politische Karriere nach dem Krieg anstreben, müssen zum einen den Habitus eines Berufspolitikers ausbilden, den Weber mit den Begriffen Machtgefühl und Machtinstinkt sowie Gesinnungs- und Verantwortungsethik umschrieben hat (Weber 1988, S. 545-552). Zum anderen müssen sie ihre politische Macht in legitime Herrschaftspositionen umwandeln und diese verstetigen. Für den Erfolg oder Misserfolg der politischen Karrieren von Rebellen sind zwei erklärende Variablen zentral, die im Folgenden näher erläutert werden sollen: zum einen die Kapitalausstattung der Akteure sowie zum anderen die Opportunitätsstruktur (Herzog 1975, S. 44, 51-52; Sterbling 1987, S. 280-282).

\subsection{Das Kapital der Eliten}

Der Erfolg politischer Karrieren hängt von der Ausstattung der Akteure mit spezifischen Machtmitteln oder Ressourcen ab, die in der Nachkriegszeit auf dem Spiel stehen und die Bourdieu als unterschiedliche Sorten von „Kapital“ versteht. Bourdieu unterscheidet im Wesentlichen vier Sorten von Kapital (Bourdieu 1992, S. 49-79; Schwingel 1993, S. 34-36): Das klassische ökonomische Kapital existiert in Form von Geld- oder Produktionsmitteln, das im politischen Feld auch als zentrale Ressource fungiert, um Anhänger materiell zu versorgen, Gefolgschaft zu belohnen und Privilegien zu gewähren. Darüber hinaus spielt das soziale Kapital als Summe von formellen Mitgliedschaften oder informellen Beziehungen in Organisationen oder Netzwerken eine entscheidende Rolle. Sein Umfang hängt sowohl von der Zahl der Verbindungen und der Reichweite des Netzes potenziell mobilisierbarer Beziehungen als auch von der Kapitalausstattung derjenigen ab, mit denen eine Beziehung besteht. Kulturelles Kapital, verstanden als Fähigkeiten, Fertigkeiten und Wissensformen, existiert im politischen Feld in Form von Bildung und Fachschulung, aber auch als organisationsspezifisches Dienstwissen oder Sprachkompetenz. Schließlich unterscheidet Bourdieu noch das symbolische Kapital, das die kollektive Anerkennung der anderen Kapitalformen bezeichnet. Jede als legitim anerkannte Form von Kapital ist zugleich symbolisches Kapital, auf das die Akteure im politischen Feld angewiesen sind, um ihre Macht in legitime Herrschaft zu transformieren (Bourdieu 1998, S. 108-114; Schwingel 1993, S. 79, 86-88).

Alle Akteure innerhalb eines Feldes sind mit verschiedenen Sorten und unterschiedlichem Umfang an Kapital ausgestattet. Die Kapitalausstattung der Akteure lässt sich daher differenzieren nach Gesamtvolumen und Mengenverhältnissen des Kapitals. Ferner lassen sich drei Modi der Bewegung unterscheiden: die Akkumulation von Kapital, der Tausch einer Kapitalsorte in eine andere sowie die Institutionalisierung des Kapitals, beispielsweise durch Eigentumsrechte, formalisierte Mitgliedschaften oder Bildungstitel.

Rebellen, die nach dem Krieg eine politische Karriere verfolgen, sind vor allem auf symbolisches, ökonomisches und soziales Kapital angewiesen, das über ihre Positionierung im sozialen Raum der Nachkriegsgesellschaft entscheidet. Dabei ist das im Zuge des Krieges erworbene und das in der Nachkriegsgesellschaft akkumulierte Kapital analytisch voneinander zu unterscheiden. Relevant für die Nachkriegskarrieren von Rebellen ist überwiegend Kapital, das nach dem Kon- 
flikt gewonnen wird. In welcher Kombination und Gewichtung diese Machtmittel eingesetzt werden können, hängt zudem von weiteren Bedingungen ab, welche die Verwertungs- und Konvertibilitätsmöglichkeiten des Kapitals festlegen. Sie bestimmen in welcher Weise das Kapital in Handlungschancen umgesetzt werden kann (Sterbling 1987, S. 280-282). Diese strukturell vorgeprägten Möglichkeiten lassen sich als „Opportunitätsstrukturen“ (Merton 1996, S. 153-161) fassen.

\subsection{Die Opportunitätsstruktur}

In der soziologischen Handlungstheorie bezeichnet der Begriff der Opportunitätsstruktur für Akteure eine objektive Handlungssituation, von deren Gestalt der Handlungserfolg abhängt. Robert Merton definiert dies wie folgt: „Opportunity structure designates the scale and distribution of conditions that provide various probabilities for individuals and groups to achieve specifiable outcomes" (Merton 1996, S. 153, Hervorh. i. O.). Opportunitäten lassen sich demzufolge als Handlungsoptionen und -chancen verstehen, die durch Strukturen geformt werden. Diese Opportunitätsstrukturen stellen Handlungsspielräume dar, die durch Individuen oder kollektive Akteure nicht kurzfristig verändert werden können. Erklärungen durch den Hinweis auf Opportunitätsstrukturen fokussieren auf Optionen und Erfolgschancen des Handelns jenseits spezifischer Akteursqualitäten. Sie lenken jedoch auch den Blick auf erfolglose Handlungen, die wegen ihrer Strukturabhängigkeit scheitern. Der Zugang zu Opportunitäten ist nicht gleich verteilt, sondern besteht abhängig von der Position in der Sozialstruktur und ist daher schicht- oder milieuspezifisch. Die Struktur der Opportunitäten unterliegt zugleich einem sozialen Wandel, in dessen Folge sich die Handlungsspielräume für Akteure erweitern oder verengen können: „[R]estricted opportunities become constraints; fewer constraints enlarge opportunities“ (Blau 1990, S. 145). Handlungen von Akteuren können darüber hinaus Effekte generieren, die den Möglichkeitsraum anderer Akteure einschränken, sodass sich die strukturellen Voraussetzungen für Anschlusshandlungen verändern und neue Rigiditäten oder Flexibilitäten entstehen (Mackert 2010, S. 409-410).

Die Beendigung eines Krieges stellt immer eine Umbruchsituation oder „critical juncture “ dar, die für nicht integrierte Gruppen einen Zugang zu Opportunitäten schafft und Aufstiegschancen eröffnet. Auch für Nachkriegsgesellschaften lässt sich daher von einer Opportunitätsstruktur sprechen, welche die Strategien individueller und kollektiver Akteure beeinflusst und ihnen ermöglicht, Machtmittel für die Erlangung von Herrschaftspositionen einzusetzen. Rebellen müssen daher ihr Kapital für die Verfolgung politischer Karrieren im Rahmen einer spezifischen Opportunitätsstruktur mobilisieren und nutzen. Diese Struktur wird von lokalen und globalen Bedingungen geformt, wobei sich drei Dimensionen unterscheiden lassen: Kriegsbeendigung, Demokratisierung und Internationalisierung.

Die Art der Kriegsbeendigung entscheidet wesentlich über Chancen von Rebellen in Nachkriegssituationen zu reüssieren. Bei der Neuordnung politischer Herrschaft nach einem Krieg treffen in der Regel Herausforderer und Etablierte eines politischen Regimes aufeinander (Tilly 2006, S. 19). Entscheidend für die Opportunitäten von Rebellen ist das Ausmaß, in dem alte Eliten ihre formellen und in- 
formellen Machtpositionen räumen müssen und damit den Weg für Gegeneliten und neue Akteursfigurationen frei machen. Die Kriegsbeendigung in Form eines Rebellensieges eröffnet größere Möglichkeiten, die Hegemonie der alten Regimeeliten $\mathrm{zu}$ brechen und eine umfassendere Elitenzirkulation anzustoßen. Wo Kriege durch Verhandlungslösungen beendet werden, sind dagegen Arrangements der Machtteilung häufig, die den Charakter großer Koalitionen haben und die für etablierte Machthaber größere Chancen des Verbleibens in Elitepositionen bergen (Roeder u. Rothchild 2005; Sriram 2008). Nachkriegssituationen können daher von einer Auflösung, aber auch von einer weitgehenden Persistenz des bestehenden politischen Regimes gekennzeichnet sein, gemessen an der horizontalen Breite und vertikalen Tiefe der neu zu besetzenden Elitepositionen. ${ }^{8}$ Die Kriegsbeendigung entscheidet über die Chancen von Rebellen Zugang zu staatlichen Positionen zu erlangen. Kriegsbeendigungen eröffnen Opportunitäten für siegreiche Rebellen, aber sie bereiten eher Zwänge für jene, die sich in Verhandlungslösungen fügen müssen.

Das zweite Element der Opportunitätsstruktur ist die Demokratisierung. Hierunter fallen alle Chancen und Zwänge der Generierung politischer Unterstützung, die Rebellen nach dem Krieg erlangen müssen. Dies betrifft sowohl die Beziehungen zwischen Elite und Masse als auch Beziehungen innerhalb der Elite. Entscheidend sind hier kompetitive Wahlen, in denen Rebellen um Wählerstimmen konkurrieren und politische Bündnisse eingehen müssen. Wahlen schaffen Opportunitäten für diejenigen Rebellen, die bereits im Krieg eine große und aktive Unterstützerbasis hatten, aber sie bedeuten Zwänge für jene, die eine solche Basis nicht hatten. Rebellen mit einer größeren Unterstützung in der Bevölkerung und der Verfügung über organisatorische Ressourcen wie etwa bereits etablierte politische Parteien können leichter Stimmen jenseits ihrer ursprünglichen Hochburgen erlangen und charismatische, klientelistische oder programmatische Beziehungen zu ihrer Wählerschaft ausbilden (Kitschelt 2000; Putnam 1976, S. 143-160). Eine große Wählerbasis oder die Führung einer Partei, die breite Unterstützung mobilisieren kann, erleichtert wiederum die Ausbildung von Kooperationsmustern zu anderen Teileliten. Denn als etablierter Akteur mit einer Machtbasis sind ehemalige Rebellen potenzielle strategische Partner für andere Elitefraktionen, die zur Sicherung ihrer hegemonialen Stellung auf Bündnisse angewiesen sind (Schlichte 2004, S. 197).

Die Opportunitätsstruktur der Eliten ist nicht zuletzt von der Internationalisierung des politischen Feldes durch die Intervention externer Akteure geprägt. Keine einzige Nachkriegssituation lässt sich mehr als lokales Geschehen begreifen. Das Ausmaß der Internationalisierung variiert aber erheblich und reicht von externen Vermittlungsbemühungen bei Friedensabkommen über die Durchsetzung von Formen der Machtteilung bis zu Protektoraten, in denen internationale Akteure über die Zusammensetzung von Regierungen mitentscheiden (Sisk 1996, S. 87-118; Doyle u. Sambanis 2006, S. 319-332). Externe Akteure können die Handlungs-

8 Die verfügbare empirische Evidenz deutet indes darauf hin, dass innerstaatliche Kriege auch im Fall eines Rebellensieges nur selten zu totalen Elitenumwälzungen führen. Siehe zum Zusammenhang von politischen Krisen und Elitenwandel Dogan u. Higley (1998). 
spielräume von Rebellen mit Instrumenten wie internationaler Strafverfolgung, Sanktionen oder Praktiken des „Naming and Shaming“ beschränken. Da intervenierende Akteure aber auch versuchen, kurzfristig Sicherheit zu schaffen oder die Regeln der politischen Inklusion zu beeinflussen, erwachsen aus der Internationalisierung für Rebellen auch Chancen (Manning 2007). Hierzu zählt die Unterstützung einflussreicher Warlords, die Stabilität versprechen und sich als demokratische Reformer verstehen (Barnett u. Zürcher 2009; Hensell u. Gerdes 2012). Rebellen können dabei politische Allianzen mit externen Akteuren schmieden und sich international legitimieren. Die Internationalisierung eröffnet daher denjenigen Rebellen Chancen, die als offizielle Verhandlungspartner akzeptiert werden, aber sie beschränkt jene, die international sanktioniert werden.

\subsection{Die Positionen in Nachkriegskarrieren}

Rebellen brauchen für ihre Karrieren eine Ausstattung mit unterschiedlichen Sorten von Kapital, aber die Möglichkeiten dieses Kapital zu akkumulieren, zu tauschen und zu institutionalisieren werden von der Opportunitätsstruktur beeinflusst. Dieses Zusammenwirken lässt sich weiter anhand eines Stufenmodells präzisieren, das es erlaubt, einzelne Positionen politischer Karrieren zu unterscheiden und diesbezügliche Verläufe zu analysieren. Eine Karriere verstehen wir dabei als Sequenz von Postionen, die sukzessiv durchlaufen werden und jeweils weitere Chancen des Aufstiegs beeinflussen (Herzog 1975, S. 39-52). Wir unterscheiden die Ausgangsposition am Ende des Krieges, die Übergangsposition in einer Transitionsphase und die Eliteposition im Staat. Diesbezüglich leiten wir folgende These ab: Rebellenkarrieren sind in erster Linie von Machtmitteln abhängig, die nach dem Konflikt gewonnen werden. Wichtig ist dabei zunächst ökonomisches Kapital. Rebellen müssen einen frühen Zugang zum Staat und dessen Einkommensquellen erlangen, denn Vorteile wachsen überproportional für jene, die mit Vorteilen starten (Manning 2008, S. 153). Entscheidend für den langfristigen Erfolg, gemessen an der dauerhaften Einnahme einer Eliteposition, ist jedoch die Konversion des ökonomischen in soziales Kapital. Rebellen müssen neue vertikale und horizontale Bündnisse schmieden - durch den Aufbau einer erweiterten Klientelbasis und durch Integration in die Machtelite. ${ }^{9}$

In der Ausgangsposition am Ende des Krieges verfügen Rebellen über diverse Ressourcen wie militärische Macht, organisatorische Kapazitäten, Unterstützung in der Bevölkerung oder externe Patronage. Werden Rebellen als legitimer politischer Akteur offiziell anerkannt, dann erlangen all diese Ressourcen eine symboli-

9 Die naheliegende Gegenthese würde die Bedeutung und Kontinuität der bereits im Krieg erlangten Machtmittel betonen. Wir halten diese Gegenthese für weniger plausibel und sehen zugleich ein Problem in ihrer Überprüfung. Hier müssten größere Zeiträume unter Einbeziehung der Kriegsphase abgedeckt werden. Dabei käme es darauf an, die von Rebellen im Krieg akkumulierten ökonomischen, organisatorischen oder symbolischen Machtmittel zu erfassen (Schlichte 2004), um dann in genaueren verlaufssoziologischen Analysen ihre Bedeutung für die Nachkriegskarrieren von Rebellen zu untersuchen. Ein solches Untersuchungsdesign steht zudem vor dem großen Problem, genauere empirische Daten über das Innenleben bewaffneter Gruppen und deren Anführer zu bekommen. Wir tragen dem Kontinuitätsargument jedoch insofern Rechnung, als wir die Bedeutung der bereits im Krieg erlangten Machtmittel als „symbolisches Kapital“ konzeptionalisieren (s. u.). 
sche Qualität im Sinne der kollektiven Anerkennung durch andere Akteure wie internationale Organisationen, Staaten, die bekämpfte Regierung oder die lokale Bevölkerung. Sie werden damit symbolisches Kapital (Bourdieu 1998, S. 108114). Das symbolische Kapital ist die zentrale Inklusionsvoraussetzung, denn es stellt die Berechtigung auf Teilhabe an der Herrschaft in der Nachkriegsordnung dar. Je mehr symbolisches Kapital, desto größer der legitime Anspruch auf politische Positionen und damit die Machstellung der Rebellen. Der Umfang dieses Kapitals ist jedoch davon abhängig, ob Rebellen international unterstützt oder sanktioniert werden und ob sie einen Sieg erringen oder einer Verhandlungslösung zustimmen müssen. Die von der Internationalisierung und Kriegsbeendigung bestimmten Opportunitäten beeinflussen die Ausstattung der Akteure mit symbolischem Kapital und bestimmt zugleich die Möglichkeiten, dieses in der Übergangsposition in andere Machtmittel zu konvertieren.

In der Übergangsposition müssen Rebellen ihre Machtbasis ausbauen und möglichst Ämter in einer Transitionsregierung erlangen, die nach dem Krieg unmittelbare Verwaltungsaufgaben übernimmt und zumeist kurz- oder mittelfristig freie Wahlen organisieren soll. Sie umfasst neben Rebellengruppen und Vertretern der alten Machthaber in der Regel auch Oppositionsparteien (Shain u. Linz 1995). Da solche Übergangsregierungen nicht selbst aus Wahlen hervorgehen, ist es für Rebellen leicht, diese Zwischenposition zu erreichen und vermittels ihres symbolischen Kapitals Ämter zu beanspruchen. Es kommt für Rebellen hier jedoch darauf an, das symbolische in ökonomisches Kapital umzumünzen. Opportunitäten für diese Konversion ergeben sich wiederum aus der Art der Kriegsbeendigung, von der die Kontinuität der alten Eliten und die Zugriffsmöglichkeiten neuer Aufsteiger auf staatliche Positionen abhängen. Rebellen brauchen einen frühen Zugang zu den Ressourcen des Staates, um gegenüber ihren Anhängern Versprechen der Patronage und Distribution glaubhaft zu machen. Diese Patronagemacht steigert die Chancen des Vorrückens auf eine Eliteposition.

In der Eliteposition müssen Rebellen ihre Macht konsolidieren und reproduzieren. Entscheidend hier ist der Tausch von ökonomischem in soziales Kapital. Die Opportunitäten hierfür werden vor allem durch die Demokratisierung bestimmt. Ehemalige Rebellenführer müssen Wahlämter jenseits ihrer ursprünglichen Hochburgen erlangen. Eine größere Unterstützerbasis macht es Rebellen leichter, soziales Beziehungskapital über alte Kameradschaftsbeziehungen hinaus zu akkumulieren und in politischen Parteien zu institutionalisieren, die über Mitglieder und Aktivisten verfügen und die Chancen auf Wahlämter steigern (Manning 2008, S. 145). Rebellenführer müssen sich insbesondere als Patron etablieren und soziales Kapital in klientelistischen Netzwerken akkumulieren, in denen Meinungsführer und grassroots Stimmen im Austausch für materielle Vorteile und Privilegien liefern (Kitschelt u. Wilkinson 2007). Je größer die Klientel und der Erfolg in Wahlen, desto stabiler die Position des Patron und desto leichter ist es für Rebellen, soziales Beziehungskapital auch zu alten und neuen Vertretern der Machtelite aufzubauen, die ebenfalls auf Bündnispartner angewiesen sind. Dies erlaubt es Rebellen sich in strategische Gruppen zu integrieren, die ein gemeinsames Interesse an dem Erhalt und der Ausweitung von Aneignungschancen entwickeln (Evers u. Schiel 1988). 
Die politischen Karrieren von ehemaligen Rebellen in der Nachkriegsgesellschaft lassen sich als abgestufte Prozesse verstehen. Sie setzten sich aus einzelnen Positionen zusammen, in denen die Kapitalausstattung der Akteure und die Opportunitätsstruktur des sozialen Raumes jeweils zusammenwirken. Wenn es Rebellen gelingt, eine gute Ausgangsposition aufgrund ihres symbolischen Kapitals zu erlangen, dann ist es wahrscheinlicher, dass sie in einer Zwischenregierung eine gute Übergangsposition beziehen, die ihnen frühen Zugang zu staatlichen Ressourcen verschafft und es erlaubt, ökonomisches Kapital zu akkumulieren, was wiederum die Chancen erhöht, dieses in soziales Kapital zu tauschen und damit in der Eliteposition neue Bündnisse und Allianzen zu bilden. Dieser Prozess unterliegt jedoch keiner Pfadabhängigkeit. Die Ausgangskonstellation bei Kriegsende macht späte Entwicklungen nur mehr oder weniger wahrscheinlich, ohne sie zu determinieren.

Um unsere Frage nach dem Erfolg und Misserfolg von Rebellen nach dem Krieg zu beantworten, sollen im Folgenden unterschiedliche Rebellenkarrieren in zwei Fallstudien zu Liberia und zum Kosovo mit Hilfe des theoretischen Rahmens erklärt werden. Beide Fälle weisen eine internationalisierte Kriegsbeendigung mit anschließender Demokratisierung auf. In beiden Fällen verfügten Rebellen am Ende des Krieges über gute Machtpositionen und schlugen politische Nachkriegskarrieren ein, allerdings mit unterschiedlichem Erfolg. Während sie im Kosovo dauerhaft zahlreiche Führungspositionen einnehmen konnten, gelang ihnen in Liberia kein vergleichbarer Aufstieg. Die Fälle Kosovo und Liberia weisen ähnliche Rahmenbedingungen, aber eine Varianz der abhängigen Variablen auf und ermöglichen daher einen ersten Plausibilitätstest unserer Annahmen.

Der methodische Ansatz in den Fallstudien folgt der Logik einer qualitativen verlaufssoziologischen Analyse mit dem Ziel, die Entwicklungsverläufe von Karrieren zu erfassen. Dies muss jedoch aus Platzgründen in stark verdichteter Form erfolgen. Die Fallstudien rekonstruieren die Nachkriegskarrieren ehemaliger Rebellen anhand des obigen skizzierten Modells, dessen drei Stufen zugleich als Analyseraster dienen. Wir fokussieren uns dabei auf Individuen und Personengruppen. Dabei soll der Zusammenhang von Kapitalakkumulation und verfügbaren Chancen sowie die daraus resultierenden Konsequenzen auf den jeweiligen Stufen verdeutlicht werden. Sofern nicht anders angegeben basiert das Datenmaterial auf der Auswertung biografischen Materials und halbstrukturierten Leitfadeninterviews mit ehemaligen Mitgliedern der Rebellen, Vertretern der Elite und politischen Beobachtern im Verlauf von Feldforschungen der Autoren in beiden Ländern in den Jahren 2011 und 2012.

\section{Die Transformation von Gewaltakteuren in Liberia und im Kosovo}

\subsection{Der langsame Abstieg der Rebellen in Liberia}

Zwei voneinander zu unterscheidende Kriege haben in Liberia verschiedene Gewaltakteure hervorgebracht. Der erste liberianische Krieg (1989-1996) brachte Charles Taylor, Anführer der National Patriotic Front of Liberia (NPFL), an die Macht. Taylor wurde 1997 zum Präsidenten Liberias gewählt und führte bis 2003 
eine international anerkannte Regierung, in welcher die politische Nachfolgeorganisation der NPFL, die National Patriotic Party (NPP), eine zentrale Machtposition einnahm. Im zweiten liberianischen Krieg (2000-2003) wurde das TaylorRegime wiederum von den Liberians United for Reconciliation and Democracy (LURD) und der Movement for Democracy in Liberia (MODEL) gewaltsam herausgefordert. LURD und die wesentlich kleinere MODEL waren aus früheren bewaffneten Gruppen entstanden, namentlich der United Liberation Movement (ULIMO) und dem Liberian Peace Council (LPC), die bereits im ersten liberianischen Krieg gegen Taylor gekämpft hatten und nach 1997 keine Machtpositionen im neuen Regime erlangen konnten. Der zweite Krieg endete mit der Exilierung Taylors und der Etablierung einer Übergangsregierung, die Ende 2005 von einer demokratisch gewählten Regierung mit Ellen Johnson Sirleaf als Präsidentin abgelöst wurde. Trotz ihres militärischen Erfolges haben sich die Rebellen der LURD und MODEL kaum in der Nachkriegsordnung behaupten können. Zwar gewannen einzelne Führungspersönlichkeiten der LURD Parlamentssitze oder wurden auf hochrangige Posten ernannt, jedoch verloren die meisten ihre Positionen in den Folgejahren wieder. Für viele endete der Aufstieg auf der mittleren Verwaltungsebene des Staatsapparats. Noch weniger erfolgreich war die Führung der MODEL, die weitgehend marginalisiert wurde.

In der Ausgangsposition am Ende des Krieges verfügten beide Rebellengruppen über relativ gute Startbedingungen. Zwar hatten sie keinen klaren militärischen Sieg errungen, jedoch kontrollierten sie bei Kriegsende weite Teile des staatlichen Territoriums, sodass es ihnen aufgrund dieser militärischen Überlegenheit gelang, Taylor ins Exil zu zwingen. Zudem hatten sie Rückhalt in den ethnischen Minderheiten, aus denen sie sich überwiegend rekrutierten. Die LURD hatte ihre Basis vor allem unter den Mandingo, während MODEL sich fast ausschließlich aus den Krahn rekrutierte. Aufgrund der militärischen Stärke der Taylor-Regierung, dem Mangel an einer nationalen Legitimitätsbasis und internationalen Drucks beurteilten die Rebellen die Chancen auf eine Konsolidierung ihrer Macht jedoch skeptisch. Sie favorisierten daher früh eine internationale Intervention und Verhandlungslösung, um ihre Positionen abzusichern (Gerdes 2013, S. 173). So wurde der Krieg durch Friedensverhandlungen beendet, die von internationalen Akteuren initiiert und moderiert wurden. Eine zentrale Rolle spielten dabei die Economic Community of West African States (ECOWAS) und die International Contact Group for Liberia (ICGL), zu der u. a. die UN, ECOWAS, die Weltbank, die EU, die USA und Nigeria zählten. In den internationalen Friedensverhandlungen waren LURD und MODEL, die Regierung Taylor und die in einem Verhandlungsblock zusammengefassten politischen Parteien repräsentiert, wozu auch die frühere Regierungspartei NPP und weitere Parteien zählten, die Taylor nahestanden. Das international gestützte Friedensabkommen erwies sich für die Rebellen als vorteilhaft, da sie als Teil einer zu bildenden Übergangsregierung auch international anerkannt wurden und das Arrangement zudem durch eine UN-Mission (United Nations Mission in Liberia) militärisch garantiert und abgesichert wurde. Die Kriegsbeendigung und Internationalisierung eröffnete den Rebellen daher Gelegenheiten symbolisches Kapital der Anerkennung zu erlangen, das ihnen Machtchancen in der nächsten Position sicherte. 
In der Übergangsposition boten sich den Rebellen weitere Möglichkeiten ihre Stellung im Rahmen einer Zwischenregierung, der National Transitional Government of Liberia (NTGL), auszubauen. Auch hier stellte die Kriegsbeendigung das entscheidende Moment der Opportunitätsstruktur dar. So wurde den Rebellen laut Friedensabkommen knapp ein Drittel der Sitze im Übergangsparlament und knapp die Hälfte der Ministerposten zugesprochen. Auch über staatseigene Betriebe und Behörden erlangten die Rebellen Kontrolle. Die übrigen Regierungsämter wurden zwischen der ehemaligen Taylor-Regierung und einem vierten Block ziviler Parteien aufgeteilt. Die zweijährige Amtszeit der Übergangsregierung wurde von allen Beteiligten als eine "license to plunder" (Sawyer 2008, S. 180) angesehen und ging mit einer massiven privaten Aneignung auf allen Ebenen des Staates einher (ECOWAS 2005). Dies ermöglichte es den Rebellen ökonomisches Kapital zu erlangen. Begrenzt wurden diese wirtschaftlichen Chancen jedoch durch die desaströse wirtschaftliche Lage in Liberia und internationale Sanktionen. Darüber hinaus wurde die Nutzung dieser Chancen durch die Faktionalisierung beider Rebellengruppen erschwert (Gerdes 2013, S. 182-187). So nominierte LURD-Führer Sekou Conneh als Finanzminister einen Verwandten, der keine Beziehungen zu den LURD hatte. Die MODEL zugeteilten Interimspositionen wurden von ihrem Anführer, Thomas Yaya Nimely, überwiegend an außenstehende Zivilisten und Günstlinge vergeben. Lediglich vier Vertreter der MODEL, darunter ihr Anführer, nahmen hochrangige Positionen in der Übergangsregierung ein und konnten diese folglich zur Akkumulation ökonomischen Kapitals nutzen (Lidow 2011). LURD und MODEL waren zudem gezwungen, ihre Pfründe mit alten und neuen Machthabern zu teilen, die ebenfalls danach strebten, sich in der Nachkriegsgesellschaft zu positionieren und ihre Ämter für zukünftige Karrieren zu nutzen. Die Kriegsbeendigung und das hierdurch etablierte Arrangement der Machtteilung beschränkte daher auch die Chancen der Rebellen, da es zugleich den potentiellen Konkurrenten der Rebellen Aufstiegs- und Konsolidierungschancen eröffnete.

Die Chancen der Rebellen wurden ferner durch die Internationalisierung der liberianischen Nachkriegsgesellschaft begrenzt. Die ICGL hatte insbesondere die Einbindung ziviler Kräfte und Beschränkungen des passiven Wahlrechts durchgesetzt. Die Ämter des Regierungsvorsitzenden der NGTL und seines Stellvertreters wurden für Zivilpolitiker reserviert, und weder sie noch die Minister der Übergangsregierung durften bei den nächsten Parlaments- und Präsidentschaftswahlen kandidieren (Hayner 2007). Dies verringerte die Chancen der Rebellen ihr ökonomisches in soziales Kapital umzuwandeln. Daher verzichtete u. a. LURD-Anführer Conneh auf die Übernahme eines Postens in der Übergangsregierung, um in den anstehenden Wahlen 2005 kandidieren zu können.

Trotz ihrer vergleichsweise guten Stellung in der Übergangsposition gelang es den Rebellen nicht, sich dauerhaft in der Eliteposition zu behaupten. Die vom Friedensabkommen vorgesehenen demokratischen Parlaments- und Präsidentschaftswahlen beschränkten die Opportunitäten der Rebellen ganz wesentlich. Sie verfügten aufgrund ihres ethnischen Hintergrunds von Anfang an über eine begrenzte Unterstützerbasis, sodass es ihnen schwerfiel, soziales Kapital jenseits ihrer Hochburgen zu akkumulieren. Dies betraf insbesondere die LURD, die sich 
vornehmlich aus der Gruppe der Mandingo rekrutierte. Mitglieder dieser Ethnie werden in weiten Teilen der Gesellschaft als guineische Zuwanderer angesehen und aufgrund innerethnischer Konkurrenz und historisch belasteten Beziehungen $\mathrm{zu}$ anderen Gruppen sind Ressentiments gegen sie verbreitet. Zudem genossen LURD-Rebellen zwar Sympathien in der Wahlbevölkerung ihrer Ethnie, jedoch waren Mandingo nur in wenigen Wahlkreisen dominant und ihre Stimmen umkämpft. Als nachteilig erwies sich auch die begrenzte Ausstattung der Akteure mit ökonomischem Kapital. Der demokratische Wettbewerb stellte nicht nur formale Anforderungen an die Finanzmittel der Kandidaten und die finanzielle Ausstattung der Parteien, sondern erforderte auch Mittel für den Wahlkampf und Patronage, um Erwartungen auf Distribution an der Basis zu erfüllen. Das Übergangsregime hatte jedoch letztendlich nur begrenzte Akkumulationschancen eröffnet und darüber hinaus gelang es den Rebellen kaum, neue Ressourcen zu erschließen. LURD-Rebellen verfügten zwar über Beziehungen zu den relativ zahlreichen Geschäftsleuten der Mandingo. Diese hatten jedoch ein größeres strategisches Interesse an Beziehungen zu etablierten politischen Eliten als zu den Rebellen.

Der demokratische Wettbewerb in den Parlaments- und Präsidentschaftswahlen 2005 und 2011 umfasste die Konkurrenz um das Präsidentenamt sowie 30 Senatoren- und 73 Repräsentantensitze. Jedes der 15 Countys Liberias wird von zwei Senatoren vertreten, während die Repräsentanten in kleineren Wahlkreisen gewählt werden. Die Rebellen hatten bei diesen Wahlen aufgrund ihrer geringen Patronagemacht und Verfügung über Sozialkapital nur begrenzten politischen Erfolg. In den Präsidentschaftswahlen 2005 kandidierte in der ersten Runde nur der Anführer der LURD, Sekou Conneh, für das Amt des Präsidenten, wobei er lediglich 0,6 Prozent der Stimmen erhielt. Andere prominente Vertreter der beiden Rebellengruppen unterstützen von Anfang Präsidentschaftskandidaten mit zivilem Hintergrund, die keine Verbindung zu den Rebellen hatten. Die Gründung politischer Parteien verlief ebenso wenig erfolgreich. Conneh gründete 2005 die Progressive Democratic Party (PRODEM), jedoch konnte kein Bewerber der Partei in den Legislativwahlen 2005 und 2011 einen Sitz gewinnen. Thomas Nimeley, Anführer der MODEL, konnte sich als Außenminister der Übergangsregierung 2005 nicht selbst zur Wahl stellen und scheiterte mit dem Versuch, eine eigene Partei zu gründen. Ein Teil der Rebellenmitglieder integrierte sich in größere oder bereits etablierte Parteien. Neben der Unity Party (UP) von Johnson Sirleaf waren dies vor allem der Congress for Democratic Change (CDC) um George Weah, die ehemalige Taylor-Partei NPP und die All Liberia Coalition Party (ALCOP). Die ALCOP wurde Mitte der 1990er Jahre vom Warlord Alhaji Kromah als politischer Arm seiner United Liberation Movement (ULIMO), einem Vorgänger der LURD, gegründet. Insgesamt konnten Mitglieder der Rebellengruppen kaum Erfolge bei den Wahlen erzielen. Nur vier Mitglieder der LURD gewannen 2005 Legislativsitze. Von diesen vier wurden zwei in 2011 nicht wiedergewählt. Lediglich ein Kommandant der MODEL gewann in 2005 einen Legislativsitz, den er 2011 wieder verlor (Gerdes 2013, S. 214-216). Ziel der eigenständigen Kandidatur war auch nicht unbedingt die Erlangung eines Mandates. Oft sollte das Wahlergebnis vor allem den lokalen Rückhalt bzw. die Verfügung über soziales Kapital demonstrieren, um den eigenen politischen Marktwert herauszustreichen und damit die Chancen für eine spätere 
Kooptation zu erhöhen. Dies gelang vor allem einzelnen Führungspersönlichkeiten der LURD, die nach 2005 in Spitzenpositionen ernannt wurden.

Aufgrund ihrer mangelnden Repräsentation in den relevanten politischen Institutionen verfügten die Rebellen auch kaum über Chancen, sich mit anderen Gruppen strategisch zu vernetzen. Sozialkapital wird in der Elite Liberias, neben dem Parlament, vornehmlich in exklusiven sozialen Organisationen wie gesellschaftlichen Clubs, Bruderschaften und Freimaurerlogen akkumuliert. Die Spitzen dieser von der Öffentlichkeit abgeschotteten Vereinigungen bilden auch Selektorate, die Einfluss auf Ernennungen bzw. Vorschläge für Posten in Exekutive und Legislative haben. Mitglieder der LURD und insbesondere der MODEL sind deutlicher schlechter in solche Clubs integriert als ihre Rivalen aus der Taylor-Regierung und der zivilen Opposition. Die Schwierigkeiten von LURD und MODEL die Eliteposition zu erreichen bzw. sich dort zu reproduzieren ist auch auf die Stärke der alten und neuen Machtelite zurückzuführen. Eine wesentliche Rolle spielte hierbei das Netzwerk von Ellen Johnson Sirleaf, die 2005 zur Präsidentin gewählt wurde und sich 2011 erfolgreich der Wiederwahl stellte. Während die Präsidentin anfänglich die Einbindung eines breiten Spektrums von „individuals from other parties, civil society organizations, and elsewhere in Liberian society" (Sawyer 2008, S. 190) favorisierte, stellte die Bindung an die Präsidentenpartei UP zunehmend eine Bedingung für politische Karriereerfolge dar (UN Secretary-General 2012, S. 2). Johnson Sirleaf, die auf eine lange Karriere in der liberianischen Politik sowie internationalen Banken und Organisationen zurückblicken kann, verfolgt darüber hinaus eine Politik der Modernisierung des Staates. Die Reformpolitik der Präsidentin, die mit einer Betonung von Qualifikationen und fachlicher Ausbildung einhergeht, bedeutet eine relative Wertsteigerung kulturellen Kapitals in Form von Bildungsabschlüssen. Hiervon profitieren konnten vor allem jene, die langfristig zivile Karrieren in internationalen Organisationen, Wirtschaft, Zivilgesellschaft und Staat verfolgt und dabei technokratisches Fachwissen oder politische Erfahrung erlangt hatten. Kaum profitieren konnten davon die Rebellen der MODEL, die am wenigsten im gebildeten Milieu Liberias verankert sind und Schwierigkeiten hatten, qualifiziertes Personal für Posten zu stellen. Etwas besser erging es Rebellen der LURD, die in der Übergangsregierung, dank ihrer Beziehungen zu Interims-Ministern der LURD, Positionen auf den mittleren Ebenen der Administration bekleiden konnten. Die Inhaber dieser Stellen verfügten über ausreichende Kompetenzen, um die Verschlankung und Modernisierung des Staatsapparats unter Johnson Sirleaf zu überstehen (Gerdes 2013, S. 217-231).

Konsolidiert haben sich auch die Subeliten des Taylor-Regimes, die aus dem ersten Krieg hervorgegangen waren. Dies betrifft vor allem die ehemalige Regierungspartei Taylors, die NPP. Sie kann auf Regierungserfahrung und eine vergleichsweise kontinuierliche Herrschaft über Teile Liberias zurückblicken, die es ihr erlaubte, sich im nationalen Elitenmilieu zu verankern. So stellt sich die NPP heute als Amalgam von früheren NPFL-Rebellen, traditionellen Autoritäten, Nachfahren der ameriko-liberianischen Elite, Geschäftsleuten unterschiedlicher ethnischer und sozialer Provenienz und dem liberianischen Bildungsbürgertum dar. Die NPP ist kontinuierlich und vergleichsweise stark auf Ministerebene und im Parlament repräsentiert. Die Marginalisierung von LURD und MODEL in der 
Nachkriegszeit ist daher nicht zuletzt auf den Aufstieg neuer bzw. die Konsolidierung bereits etablierter Eliten in Liberia zurückzuführen.

\subsection{Der Aufstieg der UÇK im Kosovo zur Regierung}

Im Kosovo-Krieg (1998-1999) war es der albanischen Ushtria Çlirimtare ë Kosovës (Befreiungsarmee des Kosovo, UÇK) mit Hilfe der militärischen Intervention der NATO gelungen, das Milošević-Regime in der südserbischen Provinz zu stürzen. Der Exodus der serbischen Machthaber stellte die vorerst letzte Etappe in einem Prozess der überwiegend gewaltsamen Zirkulation albanischer und serbischer Eliten seit der Unabhängigkeit Serbiens vom Osmanischen Reich 1913 dar (Cohen 1989, S. 335-392). Der Kosovo-Krieg zeitigte eine massive Intervention der UN im Rahmen einer Übergangsverwaltung (UN Interim Administration in Kosovo, UNMIK), die fortan das Kosovo unter internationale Aufsicht stellte und den Wiederaufbau des Staates unterstützte. Der Krieg beförderte zugleich die Spitzen der siegreichen UÇK in staatliche Machtpositionen. Seit dem Ende des Krieges ist es den Mitgliedern der ehemaligen Rebellengruppe gelungen, sich in eine neue politische Führungsschicht zu transformieren. Angehörige der UÇK haben vier von bislang fünf Ministerpräsidenten gestellt und einen von fünf Staatspräsidenten. Mitglieder der Rebellen sind darüber hinaus in zahlreichen Positionen in der Regierung und Verwaltung vertreten.

Am Ende des Krieges verfügte die UÇK über eine sehr gute Ausgangsposition, die sie den Opportunitäten der Kriegsbeendigung verdankte. Aufgrund der militärischen und vornehmlich gegen Serbien gerichteten Intervention der NATO konnte sie den Konflikt für sich entscheiden. Im Kosovo wurde die UÇK als Gewinner des Krieges angesehen. Sie wurde als Befreierin der kosovarischen Nation von der serbischen Unterdrückung und Kämpferin für die politische Unabhängigkeit des Kosovo gefeiert. Der UÇK war es darüber hinaus gelungen, Unterstützung von Schlüsselfiguren der etablierten kosovo-albanischen Elite zu erlangen, die überwiegend eine pazifistische Position vertraten und organisierte Gewalt ausdrücklich abgelehnt hatten. Obschon die urbanen Eliten sich mehrheitlich weiterhin von der Befreiungsarmee distanzierten, erfreute sich die Rebellengruppe bei Kriegsende einer breiten Popularität in der Bevölkerung (Perritt Jr. 2008, S. 2535). Dies erlaubte es ihr, symbolisches Kapital zu akkumulieren. ${ }^{10}$ Darüber hinaus erwies sich auch die Internationalisierung des Krieges als vorteilhaft für die Chancen der Rebellen. Bereits früh hatten externe Akteure versucht, im Kosovo-Krieg zu vermitteln und dabei auch Kontakte zur UÇK geknüpft. Wurde die UÇK noch im Februar 1998 von einem Diplomaten der US-Regierung als terroristische Vereinigung bezeichnet, so traf sich bereits wenige Monate später der US-amerikani-

10 Das hier generierte symbolische Kapital versuchte die ehemalige Führung der UÇK auch in der Folgezeit für die Legitimation ihrer Elitepositionen einzusetzen. Beispielhaft hierfür sind der Kult um die Gründer und Märtyrer der UÇK sowie die Heroisierung ihres heldenhaften Kampfes, die mit einem homogenisierten Narrativ in den Medien, der Historiografie und öffentlichen Zeremonien einhergeht. Der Kult um die UÇK lässt sich als Versuch interpretieren, alternative Sichtweisen auf den Krieg auszuschließen, um dadurch kulturelle Hegemonie zu erlangen (di Lellio u. Schwandner-Sievers 2006, S. 520-524). 
sche Sondergesandte Richard Holbrooke mit UÇK-Kämpfern in einer ihrer Hochburgen im Kosovo zu Gesprächen. Damit signalisierte er die Anerkennung der Rebellen durch die USA sowie die Notwendigkeit, die UÇK bei einer politischen Lösung des Konflikts zu berücksichtigen. Durch die Teilnahme der UÇK an der internationalen Friedenskonferenz im französischen Rambouillet traten Anführer der Rebellen als Teil der kosovo-albanischen Delegation auf und erlangten dadurch auch eine offizielle Anerkennung als legitimer Verhandlungspartner und Vertreter der albanischen Bevölkerung des Kosovo. Mit ihrer Zustimmung zu den Bedingungen des Friedensvertrages, die von Serbien abgelehnt wurden, konnte sich die UÇK zudem so darstellen, als sei sie in erster Linie an einer friedlichen Lösung und nicht an einer militärischen Intervention der westlichen Staatengemeinschaft interessiert (Perritt Jr. 2008, S. 150-151). All dies trug zur Anerkennung der Befreiungsarmee als legitimer politischer Akteur bei. Die Opportunitäten der Kriegsbeendigung und Internationalisierung ermöglichten es der Guerilla daher, erhebliches symbolisches Kapital zu akkumulieren.

Aufgrund dieser Ausgangsposition gelang es der UÇK auch, auf eine gute Übergangsposition vorzurücken. Dabei stellte wiederum die Kriegsbeendigung das entscheidende Moment der Opportunitätsstruktur dar. Der Krieg hatte den Sturz des Milošević-Regimes im Kosovo und ein institutionelles Machtvakuum zur Folge, sodass sich in der unmittelbaren Nachkriegszeit für die UÇK zunächst zahlreiche Chancen ergaben, in Führungspositionen einzurücken. So gelang es der UÇK, direkt im Anschluss an die Kampfhandlungen, eine von ihnen kontrollierte Übergangsregierung zu etablieren und zentrale Positionen in den örtlichen Verwaltungen sowie in staatseigenen Betrieben zu übernehmen, sodass sie sich früh Zugang zu ökonomisch bedeutsamen Bereichen sichern konnte (Eyre u. Wittkowsky 2002, S. 20). Die von der UÇK ernannten oder protegierten Bürgermeister begannen ein System der Besteuerung von Cafés, Restaurants, Hotels, Läden und Tankstellen zu etablieren sowie weitere Ressourcen durch Schmuggelpraktiken zu generieren (Yannis 2003; Briscoe u. Price 2011, S. 13). Obschon diese Übergangsregierung nach sechs Monaten im Rahmen der UNMIK-Mission wieder aufgelöst wurde, hatte sie doch der UÇK strategische Vorteile verschafft und eine Periode der Akkumulation ökonomischen Kapitals erlaubt.

Die Internationalisierung des politischen Feldes durch die massive Intervention der UN konnte die Chancen der Rebellen nicht negativ beeinflussen. Die Machtkonsolidierung der UÇK war bereits vorangeschritten, und die UN-Präsenz im Kosovo konnte hieran später kaum etwas ändern. So wurden die inoffiziellen Bürgermeister der UÇK im Rahmen der neuen UN-kontrollieren Lokalverwaltung einfach übernommen. Darüber hinaus entschieden sich die UN dazu, mit den Anführern der UÇK eng zusammenzuarbeiten. Ziel der internationalen Akteure war es, einflussreiche Figuren wie den ehemaligen Sprecher der Rebellen, Hashim Thaçi, von Beginn an in die Strukturen der UN-kontrollierten Verwaltung zu integrieren, um Stabilität sicherzustellen (Narten 2006). Dabei gelang es Thaçi, für seine Zusammenarbeit mit den $\mathrm{UN}$ u. a. eine faktische Immunität gegen polizeiliche Ermittlungen zu erlangen. Die von den UN betriebene Politik der Elitenkooptation hat die politischen Karrieren ehemaliger UÇK-Kämpfer eher befördert als behindert (Hensell u. Gerdes 2012, S. 161-164). 
Die durch die Demokratisierung eröffneten Opportunitäten waren wiederum entscheidend für die Rebellen um in Elitepositionen vorzurücken. Aufgrund der Popularität der UÇK und ihrer zuletzt breiten Unterstützerbasis in der kosovoalbanischen Bevölkerung gelang es ehemaligen Mitgliedern der Führungsriege der Rebellengruppe leicht, auch soziales Beziehungskapital zu akkumulieren und dies in neu gegründeten politischen Parteien zu institutionalisieren. Aus der UÇK sind zwei politische Parteien hervorgegangen, die Partia Demokratike e Kosovës (Demokratische Partei des Kosovo, PDK) von Hashim Thaçi und die Aleanca për Ardhmërinë e Kosovës (Allianz für die Zukunft des Kosovo, AAK), geführt von dem ehemaligen Feldkommandeur Ramush Haradinaj. Seit den ersten Parlamentswahlen 2001 ist es beiden Nachfolgeparteien gelungen, ihren Anteil an Parlamentssitzen kontinuierlich zu steigern, sodass sie in den letzten beiden Wahlen 2007 und 2011 jeweils etwa 40 Prozent der Stimmen auf sich vereinen konnten. Der PDK und der AAK gelang es auch, klientelistische Netzwerke auszubilden. Die Parteien verfügen nicht nur über Verbindungen zu diversen kommerziellen Unternehmen, die als Sponsoren und Finanziers der Parteien agieren, sondern sie kontrollieren auch Positionen im öffentlichen Sektor, in den großen staatseigenen Unternehmen sowie in den profitablen Ministerien mit großen Etats (Briscoe u. Price 2011, S. 16-21, 26-32; Marzouk u. Collaku 2010).

Die hieraus resultierende Patronagemacht erlaubt es den Parteien neue Wählerschichten anzusprechen und somit ökonomisches in soziales Kapital zu konvertieren. Beide Parteien praktizieren eine Politik der Kooptation, um neue Wählergruppen zu gewinnen und das Image als UÇK-Nachfolgepartei abzustreifen. Populäre Persönlichkeiten, Künstler, Journalisten, Professoren und junge, gut ausgebildete Leute aus den urbanen Zentren werden gezielt rekrutiert, um den Parteien ein neues Gesicht zu geben. So hat sich insbesondere das Mitgliederprofil der PDK gewandelt, die hochrangige Regierungsposten mit Leuten besetzt hat, die keine Kriegserfahrung vorweisen können. Die Einflussmöglichkeiten dieser neuen Akteure in den Parteien bleiben jedoch begrenzt, denn die ehemaligen Rebellen haben zugleich Mechanismen der Schließung ausgebildet, um ihre Positionen zu sichern. So ist die innerparteiliche Demokratisierung schwach ausgeprägt und die Kontrolle der Parteiführer ungebrochen. Die PDK und AKK werden jeweils durch ihre Parteivorsitzenden Hashim Thaçi und Ramush Haradinaj personalisiert, die seit der Gründung der Parteien ihre Organisationen anführen, ohne interne Herausforderer agieren und faktisch alles entscheiden. Die Parteien selbst zeichnen sich durch eine hermetische und intransparente Struktur aus, und es gibt keine offene Konkurrenz für höhere Parteiposten. Von neun Mitgliedern des Parteipräsidiums der PDK sind nur zwei nicht Mitglied der UÇK gewesen. Die PDK und insbesondere die AKK haben zugleich den Charakter von Klientelparteien, die jeweils in einer Region des Kosovo stark vertreten sind, wo die Parteien ihre Wählerbasis haben (Briscoe u. Price 2011, S. 26). Hier bestehen auch direkte Kontinuitäten zu den ursprünglichen Rekrutierungsgebieten der UÇK und zur Herkunft ihrer Anführer (IKS 2011, S. 59-62).

Die Demokratisierung hat die Möglichkeiten der Rebellen jedoch auch begrenzt, denn von der Öffnung des politischen Feldes profitierten zunächst vor allem die politischen Gegner der Rebellen. Zwar war es der UÇK gelungen, die 
serbischen Machthaber im Kosovo zu vertreiben, jedoch sah sich die Guerilla im Nachkriegskosovo schnell in Konkurrenz zur alten albanischen Machtelite, deren Aufstieg sich im titoistischen Jugoslawien der 1970er Jahre vollzogen hatte (Cohen 1989, S. 348-392). Vertreter dieser urbanisierten Elite hatten in der Milošević-Ära im sogenannten kosovarischen „Schattenstaat“ die Lidhja Demokratike e Kosovës (Demokratische Liga des Kosovo, LDK) gegründet und eine pazifistische Position vertreten. Angeführt von Ibrahim Rugova hatte die LDK die Hauptvertretung der Albaner beansprucht, bis sie von dem bewaffneten Widerstand und der aufsteigenden UÇK Mitte der 1990er Jahre herausgefordert worden war. Nach dem Krieg versuchte die LDK ihre alte hegemoniale Stellung wieder einzunehmen. Im Zuge der ersten Kommunal- und Parlamentswahlen konnte sie deutliche Erfolge gegenüber den beiden Nachfolgeparteien der UÇK verbuchen. Die Konsequenz war zunächst eine ausgeprägte Konkurrenz und Polarisierung zwischen der LDK, der PDK und AAK, die auch mit einer Reihe politischer Anschläge einherging. Der Verdrängungswettbewerb unter den Parteien führte indes zu einem langsamen Abstieg der früher dominierenden LDK und dem Aufstieg der Nachfolgegruppierungen der UÇK, womit sich auch der Konflikt zwischen den Parteien zunehmend abgeschwächt hat.

Beide UÇK-Nachfolgeparteien haben schon einmal mit der LDK koaliert und gemeinsame Regierungen gebildet. Auf diese Zusammenarbeit der Eliten hatten zunächst insbesondere die UN im Rahmen ihres Protektorates einen großen Einfluss. So gelang es dem UN-Sondergesandten 2002 ein Arrangement der Machtteilung zwischen der LDK, PDK und AAK durchzusetzen. Unabhängig von den jeweils gegebenen Regierungskoalitionen bestehen jedoch auch enge Interaktionsund Kommunikationsbeziehungen innerhalb der Elite. Dies lässt sich erklären durch die soziale Homogenität der Eliten, familiäre Beziehungen sowie durch gemeinsame Erfahrungen im Studium, im Untergrund und im Exil sowie nicht zuletzt durch die geringe Bedeutung politischer Programmatiken für die Abgrenzung der Akteure. Darüber hinaus teilen die Eliten politische Grundorientierungen. Dies gilt vor allem mit Blick auf zentrale Ziele wie die Befreiung von serbischer Repression, die staatliche Unabhängigkeit des Kosovo und die Annäherung an die Europäische Union. Den ehemaligen Rebellen fiel es daher leicht, Beziehungen zu den etablierten Elitefraktionen des Kosovo aufzubauen und hier soziales Kapital $\mathrm{zu}$ akkumulieren. Das Ergebnis ist eine erfolgreiche Integration der ehemaligen Rebellen in die politische Führungsschicht im Kosovo. Da die LDK ebenso wie die PDK und AAK von einer mangelnden innerparteilichen Demokratisierung und Transparenz gekennzeichnet ist, geht die horizontale Integration der Parteieliten auch mit einer relativen sozialen Schließung der gesamten politischen Klasse einher.

\section{Fazit}

Die Integration von Rebellengruppen nach dem Krieg ist nicht auf ihre Demobilisierung oder Umwandlung in politische Parteien beschränkt. Dieser Artikel hat eine breitere elitentheoretische Perspektive eingenommen und die Transformation von Rebellen in politische Führungsgruppen analysiert. Eine solche Perspektive 
erlaubt es uns nicht nur, andere Wege der Einbindung von Rebellen zu untersuchen, sondern sie lenkt den Blick auch auf das Problem der Elitenintegration nach dem Krieg, die für die Stabilität von Nachkriegsregimen zentral ist.

Der Beitrag hat den unterschiedlichen politischen Karriereerfolg ehemaliger Rebellen in Nachkriegsgesellschaften untersucht und zu erklären versucht. Unserem theoretischen Ansatz lag die Annahme zugrunde, dass die Karrieren von Rebellen bestimmte Stufen durchlaufen. Dabei kommt es auf die Erlangung und Kombination unterschiedlicher Kapitalsorten an, wofür sich zu verschiedenen Zeitpunkten Gelegenheiten ergeben, auf welche die Akteure jedoch nur begrenzt oder keinen Einfluss haben. Unsere These hat bei der Ausgangskonstellation am Ende des Krieg angesetzt und zugleich die Bedeutung einer längerfristigen Konsolidierung betont: Die Chancen auf eine politische Nachkriegskarriere steigen für Rebellen mit einem frühen Zugang zu staatlichen Ressourcen. Entscheidend ist jedoch die Konversion dieser Ressourcen in neue Bündnisse durch den Aufbau einer erweiterten Klientelbasis und durch die Integration in die etablierte Machtelite. Die Ergebnisse aus den Fallstudien zu Liberia und Kosovo erlauben eine erste Überprüfung und Differenzierung dieser These. Während die Führungsriege der ehemaligen UÇK zum festen Bestandteil der politischen Elite wurde und zahlreiche hohe Führungspositionen übernehmen konnte, ist es in Liberia den Rebellengruppen weniger gut gelungen, sich zu konsolidieren. Nur wenige konnten für einen begrenzten Zeitraum Wahlämter erlangen und sie mussten sich überwiegend mit mittleren Führungspositionen im Staatsapparat zufrieden geben. Unserem Ansatz zufolge erklärt sich diese Varianz aus der unterschiedlichen Verfügung über Machtressourcen und strukturierte Gelegenheiten, woraus sich ungleiche Schwierigkeiten bei der späteren Konsolidierung für die Rebellen ergeben haben. Gemäß unserem Stufenschema lässt sich dies wie folgt rekonstruieren:

In der Ausgangsposition verfügten die Rebellen im Kosovo und in Liberia zunächst über ähnliche Startbedingungen. Die Kriegsbeendigung und Internationalisierung eröffnete den Rebellen gleichermaßen Machtchancen. Die durch die Intervention der NATO und UN internationalisierte Kriegsbeendigung im Kosovo machte die UÇK nicht nur zum militärischen Sieger. Sie wurde auch als Befreier von der serbischen Unterdrückung gefeiert und international als legitimer Akteur anerkannt. Die Rebellen in Liberia hatten zwar keinen Rückhalt in der zivilen Opposition und konnten keine nennenswerte internationale Unterstützung mobilisieren, sodass sie es vorzogen, einer Verhandlungslösung zuzustimmen. Jedoch gingen sie als faktische Sieger aus dem Konflikt hervor, kontrollierten bei Kriegsende weite Teile des Territoriums und hatten eine Machtbasis in ihren Herkunftsregionen. Zudem wurde die Position der liberianischen Rebellen mit der zu bildenden Übergangsregierung international anerkannt und militärisch durch die UN abgesichert. Daher verfügten sowohl die UÇK als auch LURD und MODEL über symbolisches Kapital, das ihnen Machtchancen in der nächsten Position sicherte.

In der Übergangsposition haben die Rebellen zwar ebenfalls gleichermaßen Zugang zu staatlichen Ressourcen erlangt, jedoch hat sich die Kontinuität der alten Eliten unterschiedlich ausgewirkt. Auch hier waren die Opportunitäten der Kriegsbeendigung entscheidend. Die UÇK konnte in einer von ihr kontrollierten 
Übergangsregierung das Machtvakuum unmittelbar nach dem Krieg alleine ausfüllen, sich eine Reihe von Positionen und Ressourcen aneignen und damit ökonomisches Kapital generieren. Ebenso gelang es LURD und MODEL in der liberianischen Übergangsregierung diverse Positionen einzunehmen und damit ökonomisches Kapital zu erlangen. Jedoch waren LURD und MODEL von Anfang an auf ein Arrangement der Machtteilung mit den Eliten der Taylor-Regierung und anderen zivilen Eliten angewiesen, die ihre Pfründe verteidigen und sich für die ersten Nachkriegswahlen in Stellung bringen konnten. Dies hat die weiteren Chancen der liberianischen Rebellen beeinträchtigt. Unterschiedlich hat sich hier auch die Internationalisierung auf die Chancen der Rebellen ausgewirkt. In Liberia haben extern initiierte Auflagen die Minister der Übergangsregierung von einer Kandidatur bei den nächsten Wahlen ausgeschlossen und somit die Etablierung von LURD und MODEL im politischen Feld erschwert. Dagegen haben die Rebellen im Kosovo von der grundsätzlichen Kooperationsbereitschaft der UN und der Zusammenarbeit mit internationalen Akteuren eher profitiert.

In der Eliteposition haben sich die Nachkriegskarrieren der Rebellen noch weiter auseinander entwickelt. Unter den Bedingungen der Konkurrenz um Wählerstimmen gelang es ihnen in Liberia - anders als im Kosovo - kaum sich an der Macht zu halten. Im Kosovo konnten die Nachfolgeparteien der UÇK die Chancen der Demokratisierung besser nutzen. Dank der breiten Unterstützerbasis der ehemaligen Rebellengruppe sowie der Kontrolle von Ressourcen gelang es ihnen, soziales Kapital in Patronagenetzwerken zu akkumulieren und effektive Parteien zu bilden, die für den Wahlerfolg eine wesentliche Rolle spielten. Dies hat wiederum den Ausschlag für Koalitionen mit der LDK gegeben und damit eine Integration in die albanische Elite erleichtert. Dagegen erschwerten die lokal und ethnisch eng definierte Unterstützerbasis von LURD und MODEL sowie begrenzte Ressourcen für Patronage und Wahlkampf die Erlangung sozialen Kapitals. Beiden Gruppen gelang es kaum in den Wahlen zu reüssieren und eigene Parteien zu gründen. Sie waren daher auf Posten in der Verwaltung angewiesen, die sie der Zuteilung durch die Präsidentin verdankten. Damit scheiterte auch eine Integration in die bereits etablierte Elite, die für ihren Machterhalt nicht auf eine Kooptation von Rebellenmitgliedern angewiesen war.

Der Vergleich der Nachkriegskarrieren zeigt, dass die Rebellen im Kosovo und in Liberia bei Kriegsende zwar über eine ähnliche Machtstellung und daher relativ gleiche Startbedingungen verfügten, danach jedoch unterschiedliche Wege einschlugen. Die Fallstudien stützen daher die eingangs formulierte These, wonach Rebellen für die dauerhafte Einnahme von Elitepositionen auf Machtressourcen angewiesen sind, die sich weniger einer Akkumulation im Krieg verdanken, sondern in erster Linie nach dem Konflikt generiert werden müssen. Aus dem Fallvergleich wird ersichtlich, dass die Machtstellung der Rebellen am Ende des Krieges die Chancen eines weiteren Aufstiegs nicht präfiguriert, sondern nur mehr oder weniger wahrscheinlich macht. Denn für die dauerhafte Einnahme von Elitepositionen ist die sukzessive Erlangung und Kombination unterschiedlicher Kapitalsorten entscheidend. Der frühe Zugang zu den Ressourcen des Staates ist dabei eine notwendige, aber keine hinreichende Voraussetzung für eine politische Karriere. Langfristig kommt es für Rebellen darauf an, Beziehungsmuster auszubil- 
den, die es ihnen ermöglichen, mit den Massen als auch innerhalb der politischen Klasse neue Bündnisse zu schmieden. Die Karrieren von Rebellen unterliegen daher, wie die von anderen politischen Akteuren in der Nachkriegsgesellschaft, in erster Linie der Eigengesetzlichkeit des politischen Feldes.

Dabei zeigt der Fall Liberia auch, dass Rebellen nicht notwendig politische Parteien gründen müssen, um eine politische Karriere zu verfolgen. Allerdings bestätigen die Befunde wiederum, dass die Etablierung starker Parteien eher ein Weg zur Einnahme von Elitepositionen ist (Manning 2008, S. 145). Die Befunde indizieren zudem, dass Rebellen die sich ihnen bietenden Chancen zügig ergreifen müssen, um sich strategische Vorteile gegenüber Nachzüglern zu sichern. Denn mit der Konsolidierung politischer Herrschaft verringern sich auch die Chancen der Akkommodation. Die Fallstudien verdeutlichen hier die zentrale Rolle der alten Eliten und bereits etablierten Machthaber, die aufgrund ihres Amtsinhabervorteils gegenüber Newcomern in der Regel eine bessere Position haben und damit die Aufstiegschancen der Rebellen wesentlich beeinflussen.

Aus den empirischen Befunden ergeben sich auch Schlussfolgerungen in Bezug auf die Frage des Umgangs mit nicht-staatlichen Gewaltakteuren durch internationale Akteure (Schneckener 2012, S. 473-483). Möglichkeiten, die Transformation von Rebellen in politische Führungsgruppen zu beeinflussen, ergeben sich für externe Akteure vor allem unmittelbar am Ende des Krieges. Die hier getroffenen Entscheidungen zur Inklusion oder Exklusion politischer Akteure und zur Förderung von Arrangements der Machtteilung sind für die Startbedingungen von Rebellen zentral. Für ihre weiteren Karrierewege haben internationale Akteure jedoch wenig Bedeutung, weil diese auf die Mechanismen der Selektion und Reproduktion im politischen Feld wenig Einfluss haben und nur vermittelt auf die Einhaltung demokratischer Spielregeln hinwirken können. Es sind im Kern lokale Dynamiken, die über die politischen Karrieren nach dem Krieg entscheiden (Hensell u. Gerdes 2012).

Mit der Transformation von Rebellen in politische Führungsgruppen verknüpft sich auch die Frage nach den Chancen der Stabilisierung und Demokratisierung von Nachkriegsregimen. Rebellen gelten als potenzielle Störer des Friedens, die sich nicht leicht in die Politik integrieren lassen. Die hier eingenommene elitentheoretische Perspektive und die verfügbare empirische Evidenz deuten jedoch eher auf Zwänge der Anpassung hin, sobald Gewaltakteure in einer politischen Laufbahn eine Chance zur Sicherung ihrer Machtposition sehen. Ihr Aufstieg umfasst nicht nur eine Akkulturation an die Umgangsformen und Distinktionspraktiken der politischen Klasse, die auch kulturelles Kapital erfordert und die Norbert Elias als „Verhöflichung des Kriegeradels“ (Elias 1983) analysiert hat. Ehemalige Rebellen müssen darüber hinaus mit alten und neuen Machthabern kooperieren und interagieren, um eine Marginalisierung in der Nachkriegsordnung zu vermeiden. Die Notwendigkeit der wechselseitigen Habituation und „reziproken Assimilation“ (Gramsci 1971, S. 221) innerhalb der politischen Führungsschicht steigert die Chance einer Integration der Nachkriegselite. Eine konsensual geeinte Elite gilt aus Sicht der Elitentheorie als entscheidende Voraussetzung für politische Stabilität und liberale Demokratie (Higley u. Burton 2006, S. 2). Die Elitenintegration steht jedoch immer in einem Spannungsverhältnis zur Rückbindung der Eliten 
an ihre Basis. Denn je größer die Kohäsion der Eliten, desto größer auch die Gefahr der Oligarchie. Die Integration der Nachkriegseliten kann zur Ausbildung neuer Mechanismen sozialer Schließung führen, die darauf abzielen, eine volle Demokratisierung und politische Öffnung der Nachkriegsordnung zu verhindern (Zürcher et al. 2013). Das Ergebnis sind dann häufig neue Formen autoritärer Herrschaft.

\section{Literatur}

Bakonyi, Jutta, Stephan Hensell, und Jens Siegelberg (Hrsg.). 2006. Gewaltordnungen bewaffneter Gruppen. Ökonomie und Herrschaft nichtstaatlicher Akteure in den Kriegen der Gegenwart. Baden-Baden: Nomos Verlag.

Barnett, Michael, und Christoph Zürcher. 2009. The peacebuilders's contract. How external statebuilding reinforces weak statehood. In The Dilemmas of Statebuilding. Confronting the contradictions of postwar peace operations, Hrsg. Roland Paris und Timothy D. Sisk, 23-52. London: Routledge.

Berdal, Mats, und David H. Ucko. 2009. Introduction: the political reintegration of armed groups after war. In Reintegrating Armed Groups After Conflict. Politics, Violence and Transition, Hrsg. Mats Berdal und David H. Ucko, 1-9. London: Routledge.

Blau, Peter M. 1990. Structural constraints and opportunities. Merton's contribution to general theory. In Robert Merton. Consensus and Controversy, Hrsg. Jon Clark, Celia Modgil und Sohan Modgil, 141-155. London: The Falmer Press.

Bonacker, Thorsten, Michael Daxner, Jan H. Free, und Christoph Zürcher (Hrsg.). 2010. Interventionskultur. Zur Soziologie von Interventionsgesellschaften. Wiesbaden: VSVerlag für Sozialwissenschaften.

Borchert, Jens. 2003. Die Professionalisierung der Politik. Frankfurt a. M.: Campus Verlag. Bourdieu, Pierre. 1992. Die verborgenen Mechanismen der Macht. Schriften zu Politik \& Kultur 1, Hrsg. Margareta Steinbrücke, Hamburg: VSA-Verlag.

Bourdieu, Pierre. 1998. Praktische Vernunft. Zur Theorie des Handelns (zuerst franz. 1994). Frankfurt a. M.: Suhrkamp Verlag.

Bourdieu, Pierre. 2000. Propos sur le champ politique. Lyon: Presses Univ. de Lyon.

Brewer, John D. 2010. Peace Processes. A Sociological Approach. Cambridge: Polity Press. Briscoe, Ivan, und Megan Price. 2011. Kosovo's New Map of Power Governance and Crime in the Wake of Independence. The Hague: Netherlands Institute of International Relations.

Burton, Michael G., und John Higley. 1987. Elite Settlements. American Sociological Review 52: 295-307.

Cohen, Lenard J. 1989. The Socialist Pyramid: Elites and Power in Yugoslavia. London: Tri-Service Press.

de Zeeuw, Jeroen (Hrsg.). 2008a. From Soldiers to Politicians. Transforming Rebel Movements After Civil War. Boulder: Lynne Rienner.

de Zeeuw, Jeroen. 2008b. Understanding the Political Transformation of Rebel Movements. In From Soldiers to Politicians. Transforming Rebel Movements After Civil War, Hrsg. Jeroen de Zeeuw, 1-32. Boulder: Lynne Rienner. 
di Lellio, Anna, und Stephanie Schwandner-Sievers. 2006. The Legendary Commander: the construction of an Albanian master-narrative in post-war Kosovo. Nations and $\mathrm{Na}$ tionalism 12: 513-529.

Dogan, Mattei, und John Higley (Hrsg.). 1998. Elites, Crises, and the Origins of Regimes. Lanham: Rowman \& Littlefield.

Doyle, Michael W., und Nicholas Sambanis. 2006. Making War and Building Peace. United Nations Peace Operations. Princeton: Princeton University Press.

Dudouet, Véronique, Hans J. Giessman, und Katrin Planta (Hrsg.). 2012. Post-War Security Transitions. Participatory peacebuilding after asymmetric conflicts. London: Routledge.

ECOWAS. 2005. Final Report of the ECOWAS Team of Investigators on Financial Crime in Liberia. http://www.theperspective.org/documents/final_report.pdf. Zugegriffen: 21.03.2014.

Elias, Norbert. 1983. Die höfische Gesellschaft. Frankfurt a. M.: Suhrkamp.

Evers, Hans-Dieter, und Tilman Schiel. 1988. Strategische Gruppen. Vergleichende Studien zu Staat, Bürokratie und Klassenbildung in der Dritten Welt. Berlin: Dietrich Reimer Verlag

Eyre, Danna, und Andreas Wittkowsky. 2002. Privatisation in Kosovo. The Political Economy of Property Rights and Stability in a Peace-Building Mission. Südosteuropa Mitteilungen 42: 16-35.

Field, G. Lowell, John Higley, und Michael G. Burton. 1990. A New Elite Framework for Political Sociology. Revue européenne des sciences sociales 28: 149-182.

Gerdes, Felix. 2013. Civil War and State Formation. The Political Economy of War and Peace in Liberia. Frankfurt a.M: Campus Verlag.

Gramsci, Antonio, 1971: Selections from the Prison Notebooks. London: Lawrence \& Wishart.

Hartmann, Michael. 2008. Elitesoziologie. Eine Einführung. Frankfurt a. M.: Campus Verlag.

Hayner, Priscilla. 2007. Negotiating Peace in Liberia. Preserving the Possibility for Justice. HD Report November, Geneva: International Center for Transitional Justice. http://www. ictj.org/static/Africa/Liberia/HaynerLiberia1207.eng.pdf. Zugegriffen: 08.01.2010.

Hensell, Stephan, und Felix Gerdes. 2012. Elites and International Actors in Post-War Societies. The Limits of Intervention. International Peacekeeping 19, 154-169.

Herzog, Dietrich. 1975. Politische Karrieren. Selektion und Professionalisierung politischer Führungsgruppen. Opladen: Westdeutscher Verlag.

Higley, John, und Michael Burton. 2006. Elite Foundations of Liberal Democracy. Lanham: Rowman \& Littlefield.

IKS. 2011. A Power Primer. A Handbook to Politics, People and Parties in Kosovo. Prishtina: Kosovar Stability Initiative.

Kitschelt, Herbert. 2000. Linkages between Citizens and Politicians in Democratic Polities. Comparative Political Studies 33: 845-879.

Kitschelt, Herbert, und Steven I. Wilkinson (Hrsg.). 2007. Patrons, Clients, and Policies. Patterns of Democratic Accountability and Political Competition. Cambridge: Cambridge University Press.

Lidow, Nicholai Hart. 2011. Violent Order: Rebel Organization and Liberia's Civil War. Doktorarbeit, Stanford University. http://purl.stanford.edu/cd347ss0802. Zugegriffen: 02.10.2013. 
Mackert, Jürgen. 2010. Opportunitätsstrukturen und Lebenschancen. Berliner Journal für Soziologie 20: 401-420.

Malešeivić, Siniša. 2010. The Sociology of War and Violence. Cambridge: Cambridge University Press.

Malthaner, Stefan. 2007. The „Armed Groups Database“. Aims, Sources, and Methodology. Junior Research Group "Micropolitics of Armed Groups”, Working Papers 2/2007. Berlin: Humboldt-Universität Berlin.

Manning, Carrie. 2007. Interim Governments and the Construction of Political Elites. In Interim Governments. Institutional Bridges to Peace and Democracy? Hrsg. Karen Guttieri und Jessica Piombo, 53-72. Washington D. C.: United States Institute of Peace Press.

Manning, Carrie. 2008. The Making of Democrats. Elections and Party Development in Postwar Bosnia, El Salvador, and Mozambique. New York: Palgrave Macmillan.

Marzouk, Lawrence, und Petrit Collaku. 2010. Kosovo Minister's Friends Flourish from Road Bonanza. Prishtina Insight 8-22.04.2010: 1-3.

Merton, Robert K. 1996. On Social Structure and Science, Hrsg. Piotr Sztompka. Chicago: University of Chicago Press.

Muggah, Robert (Hrsg.). 2009. Security and Post-Conflict Reconstruction. Dealing with fighters in the aftermath of war. London: Routledge.

Narten, Jens. 2006. Building Local Institutions and Parliamentarianism in Post-war Kosovo. A Review of Joint Efforts by the UN and OSCE from 1999-2006. Helsinki Monitor 17: $144-159$.

Perritt Jr., Henry H. 2008. Kosovo Liberation Army. The inside story of an insurgency. Urbana: University of Illinois Press.

Putnam, Robert D. 1976. The Comparative Study of Political Elites. Englewood Cliffs: Prentice-Hall.

Roeder, Philip G., und Donald Rothchild (Hrsg.). 2005. Sustainable Peace. Power and Democracy after Civil Wars. Ithaca: Cornell University Press.

Sawyer, Amos. 2008. Emerging Patterns in Liberia's Post-conflict Politics: Observations From the 2005 Elections. African Affairs 107: 177-199.

Schlichte, Klaus. 2004. Krieg und bewaffneter Konflikt als sozialer Raum. In Kriege als (Über)Lebenswelten. Schattenglobalisierung, Kriegsökonomien und Inseln der Zivilität, Hrsg. Sabine Kurtenbach und Peter Lock, 184-199. Bonn: Dietz Verlag.

Schlichte, Klaus. 2009. In the Shadow of Violence. The Politics of Armed Groups. Frankfurt a. M: Campus Verlag.

Schneckener, Ulrich. 2012. Die Rolle nicht-staatlicher Gewaltakteure in der internationalen Politik. In Einführung in die Internationale Politik. Studienbuch, Hrsg. Michael Staack, 455-484. München: Oldenbourg Verlag.

Schwingel, Markus. 1993. Analytik der Kämpfe. Macht und Herrschaft in der Soziologie Bourdieus. Hamburg: Argument-Verlag.

Serwer, Daniel, und Patricia Thomson. 2007. A Framework for Success. International Intervention in Societies Emerging from Conflict. In Leashing the Dogs of War. Conflict Management in a Divided World, Hrsg. Chester A. Crocker, Fen Osler Hampson und Pamela Aall, 369-387. Washington D. C.: United States Institute of Peace.

Shain, Yossi, und Juan J. Linz. 1995. Between states. Interim governments and democratic transitions. Cambridge: Cambridge University Press. 
Sisk, Timothy D. 1996. Power Sharing and International Mediation in Ethnic Conflict. Washington D. C.: United States Institute of Peace.

Söderberg Kovacs, Mimmi. 2007. From Rebellion to Politics. The Transformation of Rebel Groups to Political Parties in Civil War Peace Processes. Department of Peace and Conflict Research, Report 77. Uppsala: Uppsala University.

Sorokin, Pitirim. 1927. Social Mobility. New York, London: Harper.

Springer, Natalia. 2007. Die Deaktivierung des Krieges. Zur Demobilisierung von Gesellschaften nach Bürgerkriegen. Baden-Baden: Nomos Verlag.

Sriram, Chandra Lekha. 2008. Peace as governance. Power-sharing, armed groups and contemporary peace negotiations. Basingstoke: Palgrave Macmillan.

Sterbling, Anton. 1987. Eliten im Modernisierungsprozess. Ein Theoriebeitrag zur vergleichenden Strukturanalyse. Hamburg: Universität Der Bundeswehr.

Tilly, Charles. 2006. Regimes and Repertoires. Chicago: University of Chicago Press.

UN Secretary-General. 2012. Twenty-Fourth Progress Report of the Secretary-General on the United Nations Mission in Liberia. Security Council Document S/2012/641. http:// www.un.org/Docs/sc/sgrep12.htm. Zugegriffen: 20.09.2012.

von Beyme, Klaus. 1992. Die politischen Theorien der Gegenwart. Eine Einführung. Opladen: Westdeutscher Verlag.

Weber, Max. 1988. Gesammelte Politische Schriften. Tübingen: Mohr Siebeck Verlag.

Yannis, Alexandros. 2003. Kosovo. The Political Economy of Conflict and Peacebuilding. In The Political Economy of Armed Conflict. Beyond Greed and Grievance, Hrsg. Karen Ballentine und Jake Sherman, 167-95. Boulder: Lynne Rienner.

Zürcher, Christoph, Carrie Manning, Kristie D. Evenson, Rachel Hayman, Sarah Riese, und Nora Roehner. 2013. Costly Democracy. Peacebuilding and Democratization after War. Stanford: Stanford University Press.

\section{Autorenangaben}

Dr. Stephan Hensell, Universität Hamburg, Institut für Politikwissenschaft / AKUF, Allende-Platz 1, 20146 Hamburg, stephan.hensell@uni-hamburg.de

Dr. Felix Gerdes, Universität Hamburg, Institut für Politikwissenschaft / AKUF, Allende-Platz 1, 20146 Hamburg, felix.gerdes@uni-hamburg.de 